\title{
Feature Preserving Point Set Surfaces based on Non-Linear Kernel Regression
}

\author{
A. C. Öztireli ${ }^{1}$, G. Guennebaud ${ }^{2,3}$ and M. Gross ${ }^{1}$ \\ ${ }^{1}$ ETH Zurich, Switzerland $\quad-\quad{ }^{2}$ National Research Council, Italy $\quad-\quad{ }^{3}$ INRIA Bordeaux, France
}

\begin{abstract}
Moving least squares (MLS) is a very attractive tool to design effective meshless surface representations. However, as long as approximations are performed in a least square sense, the resulting definitions remain sensitive to outliers, and smooth-out small or sharp features. In this paper, we address these major issues, and present a novel point based surface definition combining the simplicity of implicit MLS surfaces [SOSO4, Kol05] with the strength of robust statistics. To reach this new definition, we review MLS surfaces in terms of local kernel regression, opening the doors to a vast and well established literature from which we utilize robust kernel regression. Our novel representation can handle sparse sampling, generates a continuous surface better preserving fine details, and can naturally handle any kind of sharp features with controllable sharpness. Finally, it combines ease of implementation with performance competing with other non-robust approaches.
\end{abstract}

\section{Introduction}

Central to most point based graphic techniques [GP07] is the definition of an efficient and effective surface representation approximating or interpolating the input points. In this context, moving least squares (MLS) has become a popular tool on which several meshless surface definitions have been built. While initial approaches [ABCO* 03$]$ based on Levin's projection operator [Lev03] were relatively expensive to compute, significant efforts have been devoted to design simpler and more efficient methods [AK04a, SOS04]. More recently, issues such as the stability under low sampling density and real-time rendering have also been addressed [GG07, GGG08].

Since such approaches are based on least square approximations, they naturally handle uniform noise and generate smooth surfaces. On the other hand, it is well known from statistics that such approaches are highly sensitive to outliers. In the same vein, this low pass filtering process assumes data are sampled from a smooth manifold, resulting in extra smoothing (figure 2a) or even instabilities near sharp features [AK04b].

In this paper, we address these major issues via a novel MLS based surface definition. Similar to Fleishman et al.'s approach [FCOS05], we reach our goal by borrowing techniques from robust statistics [Hub04]. Indeed, as previously observed in the context of anisotropic smoothing [JDD03,

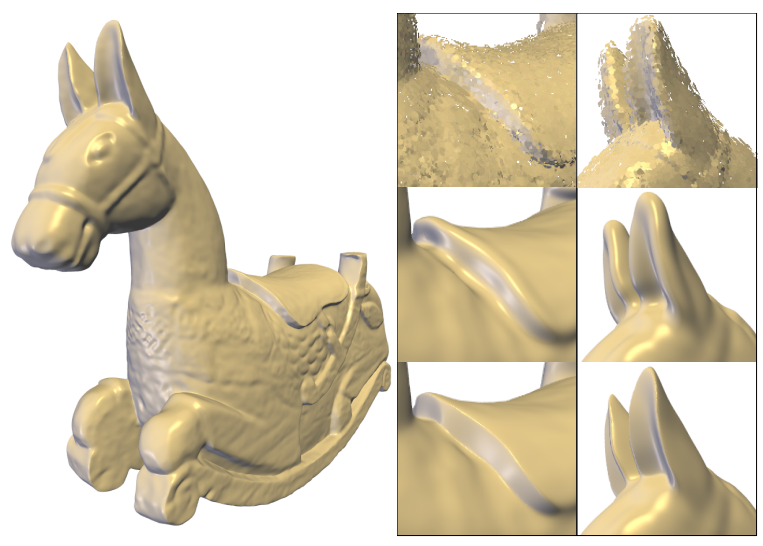

Figure 1: Left: Isidore model reconstructed using our RIMLS representation from a point set with $3 \%$ noise. Right: Closeup views of the sampling (top), and reconstructions with standard IMLS (middle) and our RIMLS (bottom).

BMdF03], samples belonging to a different smooth patch across a sharp edge can be seen as outliers in the sense of robust statistics. However, unlike Fleishman et al.'s method, our approach does not suffer from misclassification issues and generates a continuous surface while retaining all the advantages of non-robust MLS methods. To summarize, the major features of our novel MLS definition include:

- built-in handling of outliers and high frequency features, 
- controllable sharpness (globally or locally),

- stability under sparse sampling,

- ease of implementation,

- efficiency (suitable for real-time applications),

- purely local computations without preprocessing.

More precisely, as another major contribution, we show that MLS surfaces can be expressed in terms of local kernel regression (LKR) [Nad64], opening the doors to a large literature of well established techniques. In particular, we focus on implicit moving least squares (IMLS) [SOS04, Kol05], on which we build our novel representation (section 3). In a second step we show how general LKR can be properly extended with robust statistics (section 4.1). Combining IMLS with the robust LKR approach gives our novel robust IMLS surface representation (section 4.2). Finally, we also present a novel feature preserving normal smoothing procedure, tailored to the preprocessing of extremely noisy datasets.

\section{Related Work}

\section{Moving Least Squares Surfaces}

Moving least squares [She68] is a popular method for functional approximation of irregular data. Its extension to the reconstruction of manifolds has been pioneered by Levin [Lev03] and introduced to computer graphics by Alexa et al. [ABCO*03]. The surface was defined as the set of stationary points of an iterative projection operator: at each step a polynomial approximation of the local neighborhood is performed from a local planar parametrization. By omitting the polynomial fitting step, Amenta and Kil [AK04a] showed that the same surface can be defined and computed by weighted centroids and a smooth gradient field. This definition avoids the planar parametrization issues in the case of sparse sampling, and greatly simplifies the representation, especially in the presence of normals [AA04, AA07].

However, plane fit cannot perform tight approximations and becomes unstable when the sampling rate drops below some threshold [AK04b, GG07]. To overcome these limitations, Guennebaud and Gross proposed to directly fit higher order algebraic surfaces such as spheres [GG07]. Using a spherical fit with appropriate normal constraints, this approach yields a trivial and efficient closed form solution of the underlying algebraic point set surfaces (APSS) [GGG08].

With similar initial motivations, Shen et al. [SOS04] presented a radically different solution to the problem: instead of trying to fit trivariate polynomials to the data, they proposed to use standard MLS to reconstruct tangential implicit planes prescribed at each input sample position. When constant polynomials are used as the MLS basis, this method yields a simple weighted average [Kol05] that we refer to IMLS for implicit MLS. Unfortunately, without a global optimization step, this approach suffers from expanding and shrinking effects [GG07]. The method presented in this paper builds on IMLS and avoids those undesirable effects.

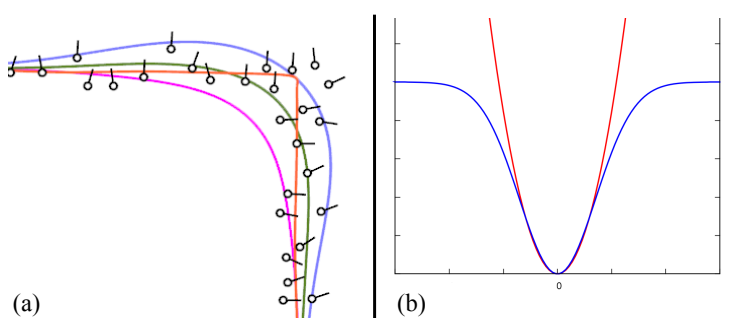

Figure 2: (a) Comparison of various MLS definitions: SPSS (purple), APSS (green), IMLS (blue), and our RIMLS (orange). (b) L2 error (red) versus the robust Welsh's function.

\section{MLS and Sharp Features}

By definition, MLS based techniques can only reconstruct smooth surfaces. To overcome this limitation, various approaches have been proposed. Some of them rely on an explicit representation of the sharp creases using either cell complexes [AA06b] or tagged point clouds [RJT* 05, GG07] to separate the input samples into different components. A more challenging task, however, is to automatically detect or enhance features present in the input point cloud.

As initially observed in the context of anisotropic smoothing [JDD03, BMdF03], samples belonging to different smooth patches across a sharp feature can be seen as outliers. This suggest the use of robust statistics both to deal with real outliers and reconstruct sharp features. Following this idea, Fleishman et al. [FCOS05] designed an iterative refitting algorithm which locally classifies the samples across discontinuities. While constituting an important progress, the method requires very dense sampling, as well as special and complex handling to locally combine the different patches making the approach relatively expensive. Furthermore, it offers only limited flexibility to the user, and the lack of global consistency of the classifications yields $C^{-1}$ discontinuities and jagged edges. While the latter limitation can be overcome using more advanced techniques [DHOS07, LCOL07], their inherent complexity makes them only suitable for surface reconstruction and not to produce an effective surface representation.

Our approach also takes inspiration from robust statistics, but in contrast to these methods, it naturally preserves any kind of high frequency features, from sharp edges to fine details, without any special handling or segmentation.

\section{Kernel Regression and MLS}

Kernel regression is a popular method in statistics to estimate the conditional expectation of a random variable [TFM07]. In this section we briefly present local kernel regression (LKR) and show the link to existing MLS surface representations. In particular we will explicitly derive Kolluri's IMLS definition in this LKR framework, that will form the basis for our new robust MLS definition in section 4. Note that a link between MLS and LKR has been very 
recently suggested only in the case of the functional setting [WSS08].

\subsection{Local Kernel Regression}

Similar to MLS, local kernel regression is a supervised regression method to approximate a function $f(\mathbf{x}): \mathbb{R}^{d} \rightarrow \mathbb{R}$ given its values $y_{i} \in \mathbb{R}$ at sampled points $\mathbf{x}_{i} \in \mathbb{R}^{d}$. The input data might be corrupted with noise such that $y_{i}=f\left(\mathbf{x}_{i}\right)+\varepsilon$, where $\varepsilon$ is a random variable with zero mean.

The essence of the method is to approximate the unknown function $f\left(\mathbf{x}_{i}\right)$ around the evaluation point $\mathbf{x}$ in terms of a Taylor expansion:

$f\left(\mathbf{x}_{i}\right) \approx f(\mathbf{x})+\left(\mathbf{x}_{i}-\mathbf{x}\right)^{T} \nabla f(\mathbf{x})+\frac{1}{2}\left(\mathbf{x}_{i}-\mathbf{x}\right)^{T} \mathbf{H} f(\mathbf{x})\left(\mathbf{x}_{i}-\mathbf{x}\right)+\ldots$

where $\mathbf{H} f(\mathbf{x})$ denotes the Hessian matrix of $f(\mathbf{x})$. The order $o$ of the expansion is defined as the number of terms used in the Taylor expansion minus one. This equation can be reformulated as a sum of inner products:

$$
f\left(\mathbf{x}_{i}\right) \approx s_{0}+\mathbf{a}_{i}^{T} \mathbf{s}_{1}+\mathbf{b}_{i}^{T} \mathbf{s}_{2}+\ldots
$$

where $\mathbf{a}_{i}=\left(\mathbf{x}_{i}-\mathbf{x}\right)$, and $\mathbf{b}_{i}=\left[\ldots\left(\mathbf{a}_{i}\right)_{j}\left(\mathbf{a}_{i}\right)_{k} \ldots\right]^{T}$ with $k \geqslant$ $j$. The local approximation is by definition more accurate nearby the point $\mathbf{x}$. This suggests the use of a weighted least squares minimization to find the unknown parameters $\mathbf{s}=$

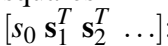

$$
\arg \min _{\mathbf{s}} \sum\left(y_{i}-\left(s_{0}+\mathbf{a}_{i}^{T} \mathbf{s}_{1}+\mathbf{b}_{i}^{T} \mathbf{s}_{2}+\ldots\right)\right)^{2} \phi_{i}(\mathbf{x})
$$

where $\phi_{i}(\mathbf{x})=\phi\left(\left\|\mathbf{x}-\mathbf{x}_{i}\right\|\right)$, and $\phi(x)$ is a symmetric and decreasing weight function giving more weight to samples near $\mathbf{x}$ in the minimization. Thus we see that equation (2) is nothing but a local least square approximation as in standard MLS.

\subsection{Deriving MLS Surfaces from LKR}

The problem of implicit surface reconstruction from point clouds consists of approximating the signed distance function to a surface given points sampled from the surface. To approximate the signed distance function using LKR, we assume that the sampled points are close to the surface so that $f\left(\mathbf{x}_{i}\right) \approx 0$ which implies to take $y_{i}=0$. In order to avoid the trivial solution $\mathbf{s}=0$, some regularization terms have to be added.

A common approach is to constrain the norm of the gradient to be one: $\|\nabla f(\mathbf{x})\|=\left\|\mathbf{s}_{1}\right\|=1$. This regularization term has been used in the context of plane [AA03] and sphere [GG07] fitting. In the presence of samples with normals, an alternative approach is to make the gradient of the implicit surface approximate the prescribed normals [AA04, GGG08].

As explained in section 2, IMLS is based on a quite different approach [SOSO4] that approximates prescribed functions rather than scalar values. However, we show that IMLS can also be derived from the aforementioned normal constraint. Indeed, given the Tailor expansion of the gradient:

$$
\nabla f\left(\mathbf{x}_{i}\right) \approx \nabla f(\mathbf{x})+\mathbf{H} f(\mathbf{x})\left(\mathbf{x}_{i}-\mathbf{x}\right)+\cdots
$$

and taking a zero order expansion give us $\nabla f\left(\mathbf{x}_{i}\right) \approx \nabla f(\mathbf{x})$. Since we assume the function $f$ approximates a signed distance field, we can set the normal constraint $\nabla f\left(\mathbf{x}_{i}\right)=\mathbf{n}_{i}$, where $\mathbf{n}_{i}$ is the normal at the sample point $\mathbf{x}_{i}$. In this case, the first order LKR minimization degenerates to a zero order LKR:

$$
\begin{gathered}
\arg \min _{s_{0}, \mathbf{s}_{1}} \sum\left(y_{i}-\left(s_{0}+\mathbf{a}_{i}^{T} \mathbf{s}_{1}\right)\right)^{2} \phi_{i}(\mathbf{x}) \\
=\arg \min _{s_{0}} \sum\left(s_{0}+\left(\mathbf{x}_{i}-\mathbf{x}\right)^{T} \mathbf{n}_{i}\right)^{2} \phi_{i}(\mathbf{x}) .
\end{gathered}
$$

Solving this minimization yields the following explicit formula for $f(\mathbf{x})$ :

$$
f(\mathbf{x})=s_{0}=\frac{\sum \mathbf{n}_{i}^{T}\left(\mathbf{x}-\mathbf{x}_{i}\right) \phi_{i}(\mathbf{x})}{\sum \phi_{i}(\mathbf{x})}
$$

which is exactly Kolluri's IMLS definition.

Due to the high similarity between MLS and LKR, the derivation of all other MLS surface definitions that fits a local implicit surface from the LKR framework is also possible. As an example, the surface definition of SPSS with plane fitting corresponds to $o=1$ with the constraint $\left\|\mathbf{s}_{1}\right\|=1$. Interpreting IMLS, and MLS surfaces in general, in terms of LKR opens many ways to improve the existing definitions and make new ones, such as using different constraints or utilizing methods and extensions developed for local kernel regression. In the scope of this work, we combine robust kernel regression techniques with IMLS to arrive at the proposed novel representation.

\section{Robust Implicit MLS}

In contrast to most previous heuristic methods for meshes or point clouds that use robust measures to handle features and outliers, we derive our new MLS surface definition by formulating it as a LKR minimization using a robust objective function. This results in a simple and theoretically sound implicit formulation of the surface.

In the following sub-sections, we first derive a robust strategy for general continuous approximation using LKR, and then we specialize it to the case of MLS surfaces and faithful sharp feature reconstruction.

\subsection{Robust Local Kernel Regression}

Standard LKR, as presented in section 3.1, assumes the data follow a smooth model with uniform noise. As a consequence, even a single outlier in the data can significantly influence the solution. Instead of relying on an extra outlier removal algorithm, robust statistics are based on statistical quantities that are not influenced by them. Some popular robust measures are least median of squares, least 
trimmed squares, and repeated median (e.g. [Mor07]). However, since the objective functions of these methods are not differentiable, they likely generate discontinuous solutions (i.e., $C^{-1}$ ) and require expensive minimizations.

Therefore, we extend LKR to robust local kernel regression (RLKR) using $\psi$-type M-Estimators, which have the significant advantage of leading to simple and efficient minimization procedures [Hub04]. Instead of the ordinary least squares criterion, $\psi$-type M-Estimators minimize a different but still differentiable objective function such that outliers are given less weight. For our problem, there are spatial and normal outliers and thus these estimators are well-suited. Using M-Estimation, the general LKR objective function of eq. (2) becomes:

$$
\arg \min _{\mathbf{s}} \sum \rho\left(y_{i}-g_{\mathbf{s}}\left(\mathbf{x}_{i}\right)\right) \phi_{i}(\mathbf{x})
$$

where $g_{\mathbf{s}}=s_{0}+\mathbf{a}_{i}^{T} \mathbf{s}_{1}+\mathbf{b}_{i}^{T} \mathbf{s}_{2}+\ldots$ corresponds to the local approximation of $f$, and $\rho$ is an arbitrary function. Assuming $\rho$ is differentiable, and taking $w(x)=\frac{d \rho}{d x} / x$, this nonlinear problem can be solved using the following Iteratively Reweighted Least Squares (IRLS) [Cle79] minimization:

$$
\mathbf{s}^{k}=\arg \min _{\mathbf{s}} \sum \phi_{i}(\mathbf{x}) w\left(r_{i}^{k-1}\right)\left(y_{i}-g_{\mathbf{s}}^{k}\left(\mathbf{x}_{i}\right)\right)^{2}
$$

where $r_{i}^{k-1}=y_{i}-g_{\mathbf{s}}^{k-1}\left(\mathbf{x}_{i}\right)$ is the $i^{t h}$ residual at the $k-1^{t h}$ iteration. Each iteration is equivalent to a weighted least square minimization. The behavior of this method depends on the choices of the function $\rho$ and initial conditions, which are discussed below.

Choice of $\rho$. To be robust to outliers, the function $\rho$ should grow slowly such that $w(x)$ decreases to zero as $x \rightarrow \infty$. Moreover, for our purposes, its continuity degree is also an important criteria. In this paper we used Welsch's function $\rho(x)=\frac{\sigma_{r}^{2}}{2}\left(1-e^{-\left(\frac{x}{\sigma_{r}}\right)^{2}}\right)$ which is $C^{\infty}$, and yields a Gaussian weight function $w(x)=e^{-\left(\frac{x}{\sigma_{r}}\right)^{2}}$. A comparison between the $L_{2}$ norm and Welsch's function is given in figure $2 \mathrm{~b}$.

Choice of the starting point. As with any non-linear optimization, a critical choice is the starting point. In the context of IRLS, it is usually recommended to initialize the iterations with another robust method such as the median or median absolute deviation. However, in the context of MLS, the continuity of the solution is of first importance. Therefore, we use solutions of the least square minimization of eq. (2) as the starting points. This has the significant advantage of leading to a continuous reconstruction at any iteration step, while being both simpler and much more efficient. In practice, this choice is equivalent to setting all the initial weights to one (i.e., $w\left(r_{i}^{0}\right)=1$ ).

\subsection{Our Robust Surface Definition}

Combining the IMLS surface definition as presented in section 3.2 with the general RLKR approach of the previous section yields a robust IMLS surface (RIMLS) defined by

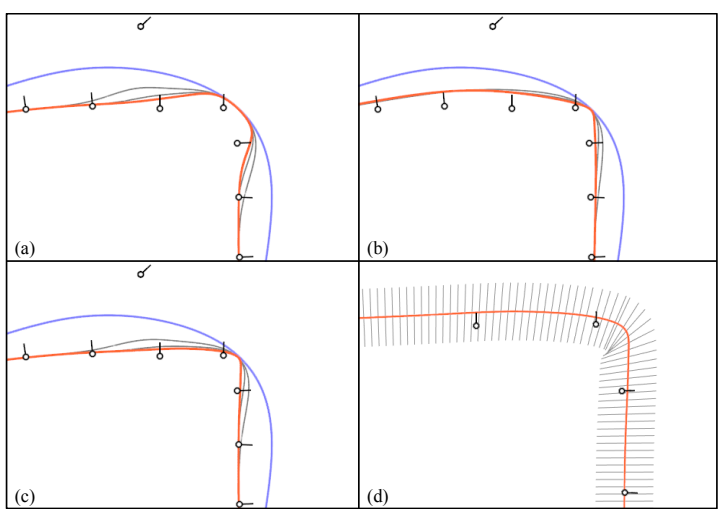

Figure 3: A corner with one outlier reconstructed using IMLS (blue) and RIMLS (orange). Grey curves show RIMLS after one and two iterations. (a) is with the residual term only, (b) is with the gradient term only, while (c) includes both terms. (d) Plot of the approximate gradient in a worst case scenario.

the following IRLS minimization:

$$
f^{k}(\mathbf{x})=\arg \min _{s_{0}} \sum\left(s_{0}+\left(\mathbf{x}_{i}-\mathbf{x}\right)^{T} \mathbf{n}_{i}\right)^{2} \phi_{i}(\mathbf{x}) w\left(r_{i}^{k-1}\right)
$$

with the residuals $r_{i}^{k-1}=f^{k-1}(\mathbf{x})-\left(\mathbf{x}-\mathbf{x}_{i}\right)^{T} \mathbf{n}_{i}$. This definition is robust to spatial outliers which is already a great improvement over the initial version.

However, our main motivation is to increase the accuracy of the reconstruction in the case of sharp features. While it is common to assume that samples belonging to a different surface patch across a discontinuity can also be interpreted as spatial outliers, it is easy to observe that this assumption does not hold nearby the actual first order discontinuity (figure 3a). We overcome this limitation by making the observation that, across a sharp feature, the actual outliers are not the samples themselves, but the sample normals. This suggests the addition of a second re-weighting term penalizing samples having normals far away from the predicted gradient of the IMLS surface. Given $\Delta \mathbf{n}_{i}^{k}=\left\|\nabla f^{k}(\mathbf{x})-\mathbf{n}_{i}\right\|$ as the measure of the difference between the predicted gradient and a sample normal, we define this new weight function $w_{n}$ as:

$$
w_{n}\left(\Delta \mathbf{n}_{i}^{k}\right)=e^{-\frac{\left(\Delta \mathbf{n}_{i}^{k}\right)^{2}}{\sigma_{n}^{2}}} .
$$

The iterative minimization of our final RIMLS definition becomes:

$$
f^{k}(\mathbf{x})=\frac{\sum \mathbf{n}_{i}^{T}\left(\mathbf{x}-\mathbf{x}_{i}\right) \phi_{i}(\mathbf{x}) w\left(r_{i}^{k-1}\right) w_{n}\left(\Delta \mathbf{n}_{i}^{k-1}\right)}{\sum \phi_{i}(\mathbf{x}) w\left(r_{i}^{k-1}\right) w_{n}\left(\Delta \mathbf{n}_{i}^{k-1}\right)}
$$

The effect of these refitting weights is illustrated in figure 3 .

Further insight into the robust weights $w_{n}$ can be gained by considering the first order regularization term $\nabla f(\mathbf{x})=n_{i}$ we used to derive the initial IMLS definition in section 3.2. The error made on this constraint is not explicitly addressed in the residuals $r_{i}^{k-1}$. In fact, it corresponds to the norm of 


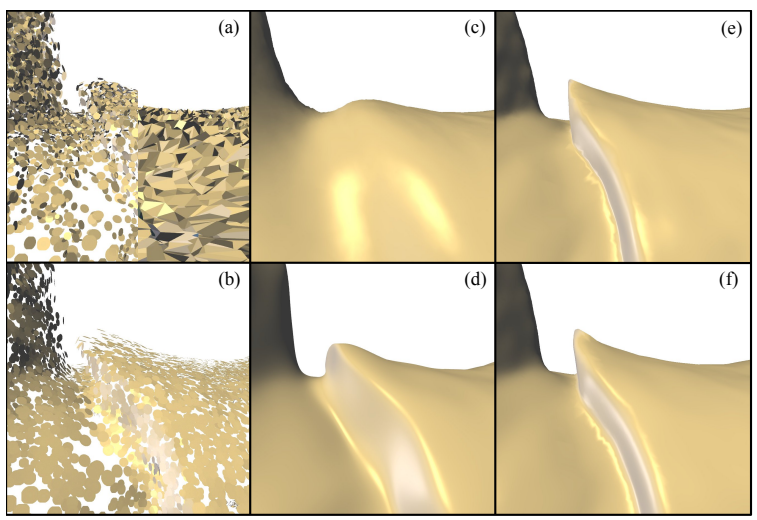

Figure 4: Illustration of the robust normal smoothing algorithm on a highly noisy input rendered as both a set of splats and a mesh (a). (b) is after the normal filtering pass. The normals are sharp near the edges. $(c)$ and $(d)$ are the final RIMLS reconstruction before and after the normal smoothing step respectively. $(e)$ and $(f)$ corresponds to our RIMLS in the presence of $25 \%$ and $40 \%$ of outliers respectively.

the residual gradient, which is equal to $\Delta \mathbf{n}_{i}^{k-1}$. Finally, it is interesting to note that equation (9) can also be seen as an iterative trilateral filtering including both zero order and first order penalizing weights.

\subsection{Computing The Surface}

The previous equation (9) entirely defines our RIMLS surface as the zero set of the signed scalar field $f$. Computing the surface, or parts of it, can therefore be achieved using either a marching cube algorithm, by casting rays, or by projecting points onto the surface. In any case, evaluating the scalar field $f(\mathbf{x})$ requires the computation of the surface gradient at each iteration.

Owing to the recursive nature of our definition, computing exact derivatives leads to rather complicated and expensive expressions. Fortunately, we show that tolerating a minor approximation, one can get pretty accurate results in a fairly easy manner. The key idea is to consider that, in the definition of the current implicit function $f^{k}(\mathbf{x})$, the refitting weights are constant values. Thus, fixing $w_{i}=$ $w\left(r_{i}^{k-1}\right) w_{n}\left(\Delta \mathbf{n}_{i}^{k-1}\right)$, the gradient $\nabla f^{k}$ becomes:

$$
\nabla f^{k}(x)=\frac{\sum w_{i} \phi_{i}(\mathbf{x}) \mathbf{n}_{i}+\sum w_{i} \nabla \phi_{i}(\mathbf{x})\left(\mathbf{n}_{i}^{T}\left(\mathbf{x}-\mathbf{x}_{i}\right)-f^{k}(x)\right)}{\sum w_{i} \phi_{i}(\mathbf{x})}
$$

The error due to this approximation is maximal when the refitting weights vary quickly, i.e., nearby very sharp edges. Figure $3 \mathrm{~d}$ shows the accuracy of this approximation in such a worst case scenario. Higher order derivatives can easily be computed in the same manner.

\subsection{Robust Normal Mollification}

Our RIMLS surface definition, like many previous ones, relies on the input surface normals. Since RIMLS embeds a low pass filter, it naturally deals very well with both spatial and normal noise. Nevertheless, when the input normals are extremely noisy it might be helpful to first filter them. In order to preserve sharp features, we accomplish this by applying the robust optimization procedure derived in the previous sections to the normal vector field. In particular, given a point $\mathbf{p}_{j}$, we define its smooth normal $\tilde{\mathbf{n}}_{j}$ as the solution of the following IRLS minimization:

$$
\mathbf{n}_{j}^{k}=\frac{\sum_{i} \phi_{i}\left(\mathbf{p}_{j}\right) w_{n}\left(\left\|\mathbf{n}_{j}^{k-1}-\mathbf{n}_{i}\right\|\right) \mathbf{n}_{i}}{\sum_{i} \phi_{i}\left(\mathbf{p}_{j}\right) w_{n}\left(\left\|\mathbf{n}_{j}^{k-1}-\mathbf{n}_{i}\right\|\right)},
$$

where $\phi_{i}$ and $w_{n}$ are defined as in the previous section. Again, a critical choice is the starting point $\mathbf{n}_{j}^{0}$. In the bilateral filtering setting, $\mathbf{n}_{j}^{0}$ would be set to the initial sample normal $\mathbf{n}_{j}$. However, it is well known that this strategy preserves outliers: for instance let's pick a sample $\mathbf{p}_{j}$ with a flipped normal, all the similarity weights $w_{n}\left(\left\|\mathbf{n}_{j}-\mathbf{n}_{i}\right\|\right)$ with $i \neq j$ will be negligible, and its normal $\mathbf{n}_{j}$ will stay unchanged. Robust starting points typically aim to overcome this issue. As a much faster approximation, we propose to initialize the iterations with the weighted mean of the neighbor normals without considering the current normal itself:

$$
\mathbf{n}_{j}^{0}=\frac{\sum_{i, i \neq j} \phi_{i}\left(\mathbf{p}_{j}\right) \mathbf{n}_{i}}{\sum_{i, i \neq j} \phi_{i}\left(\mathbf{p}_{j}\right)} .
$$

The effectiveness of our normal filtering technique is depicted in figure 4 on a highly noisy dataset. This procedure is similar to the one of Choudhury and Tumblin [CT03], and mainly differs in the fact that we are doing a full minimization and in the choice of a more robust starting point.

\section{Results}

\subsection{Implementation and Performance}

Our RIMLS algorithm is straightforward to implement: as shown by equation (9), evaluating the scalar field $f(\mathbf{x})$ is

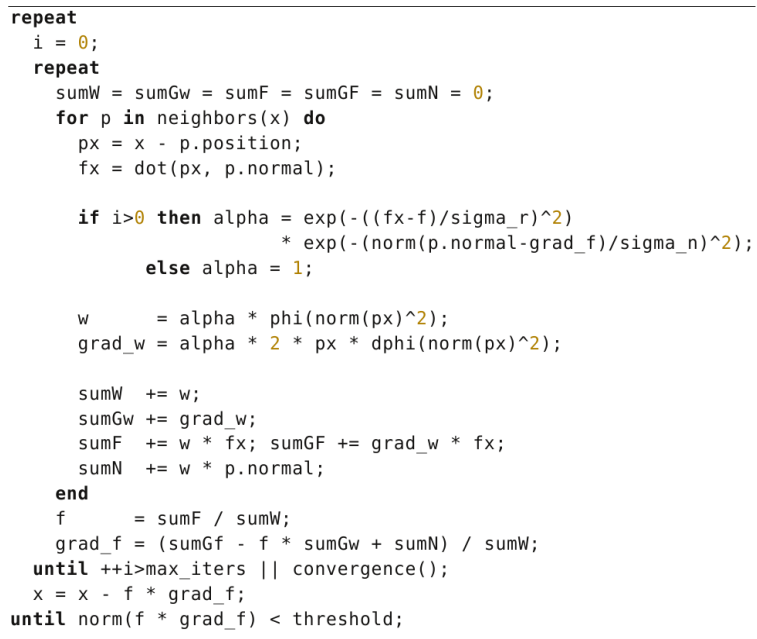

Listing 1: pseudo code of the projection procedure of a point $\mathrm{x}$ onto the RIMLS surface. 

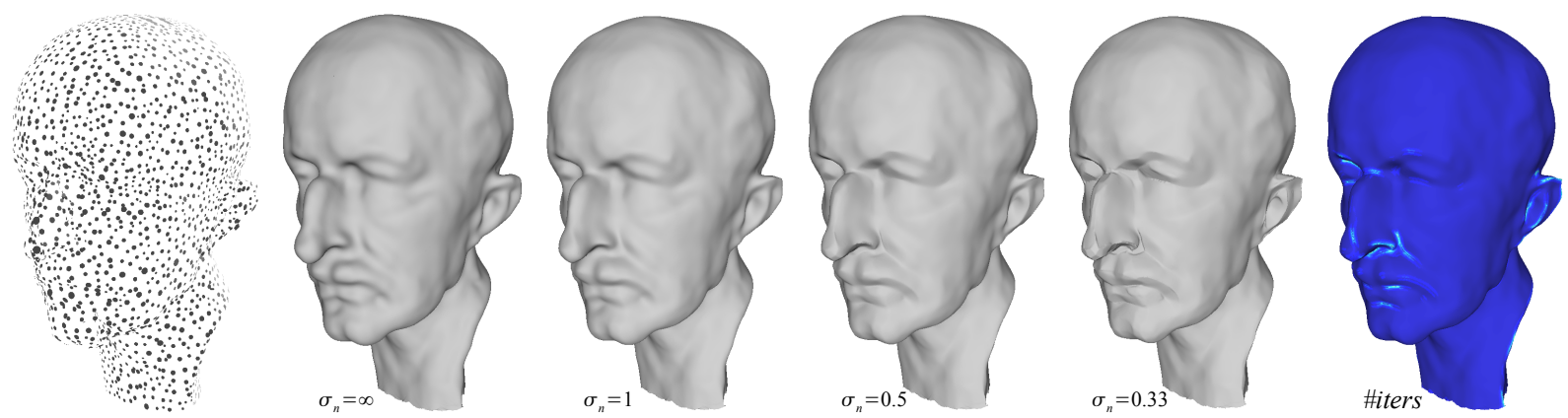

Figure 5: Illustration of the sharpness effect of the parameter $\sigma_{n}$. Note that in this example we set $\sigma_{r}=\infty$ and therefore, the case $\sigma_{n}=\infty$ corresponds to IMLS. The last picture illustrates the number of iterations to convergence for the case $\sigma_{n}=0.5$.

essentially a simple weighted average over the neighbors of $\mathbf{x}$ repeated until some condition vanishes. Listing 1 gives a pseudo code algorithm of the projection of a point onto the underlying RIMLS surface using a steepest gradient descent strategy. In the remaining parts of this section, we discuss various implementation choices and performance.

Spatial weight function. For the spatial weight function $\phi_{i}(\mathbf{x})$ we used the following $C^{3}$ continuous polynomial approximation of a Gaussian:

$$
\phi_{i}(\mathbf{x})=\left(1-\frac{\left\|\mathbf{x}-\mathbf{x}_{i}\right\|^{2}}{h_{i}^{2}}\right)^{4}
$$

where the weight radii $h_{i}$ allow to adapt to the local density. The choice of $h_{i}$ depends on the amount of noise, and typical values range from 1.4 to 4 times the local point spacing. For fast neighbor retrieval, we store the input samples in a kdtree data structure (eg, see [AA06a]).

Robust weight radii. Our robust definition introduces two additional weight radii $\sigma_{r}$ and $\sigma_{n}$. Since $\sigma_{r}$ is used to scale the residual term, which represents a difference of distance to the surface, it can be set locally as a fraction of the spatial radius $h_{i}$. Thus, the refitting weight $w$ becomes:

$$
w(x)=e^{-\left(\frac{x}{\sigma_{r} h_{i}}\right)^{2}} .
$$

This way, the choice of $\sigma_{r}$ does not depend on the object scale anymore, and it can be set once for all. In this paper we used $\sigma_{r}=0.5$.

The choice of $\sigma_{n}$ is much more subjective and depends on the desired degree of sharpness. If we assume the norm of the gradient is close to one, $\sigma_{n}$ is used to scale the difference between unit vectors, and then typical choices range from 0.5 to 1.5 . Smaller values lead to sharper results. Figures 5 and 10 illustrates this effect. We also emphasize that this sharpness parameter can be set locally for each sample, allowing the user to locally adjust the degree of sharpness, via, for instance, a painting-like tool [PKKG03].

Termination criteria. The last implementation choice concerns the stopping criterion of the iterations. One option would be to iterate until convergence is detected. We detect convergence by tracking the relative change of the refitting weights, i.e.:

$$
\max _{i}\left|\frac{w\left(r_{i}^{k}\right) w_{n}\left(\Delta \mathbf{n}_{i}^{k}\right)}{\sum w\left(r_{i}^{k}\right) w_{n}\left(\Delta \mathbf{n}_{i}^{k}\right)}-\frac{w\left(r_{i}^{k-1}\right) w_{n}\left(\Delta \mathbf{n}_{i}^{k-1}\right)}{\sum w\left(r_{i}^{k-1}\right) w_{n}\left(\Delta \mathbf{n}_{i}^{k-1}\right)}\right|<t
$$

where $t$ is a user defined threshold (eg, $t=10^{-4}$ ).

On the other hand, as seen in section 4.1, we took a special care to build RIMLS such that at any iteration it generates a reasonable and continuous surface. Therefore, one may fix a constant number of iterations while still keeping the convergence test as an early termination optimization. Note that using a fixed number of iterations, preferably small, also allows to extract a closed form formula of the implicit scalar field $f$.

As demonstrated in figures 3,5, and 12, we observed that the converge rate of our algorithm is extremely high. Not surprisingly, the number of refitting iterations for full convergence slightly increases near sharp features or high frequency details, while only one iteration is needed in smooth regions. In fact, according to our experience, in most cases the differences after one refitting iteration are seldom perceptible (eg, see figure 7).

Performance. Thanks to the very high convergence rate and simplicity of our approach, we can achieve performance of similar order as the fastest MLS definitions. In practice, the neighbor search part still remains the main computational cost. We emphasize that our RIMLS algorithm could perfectly fit into the real-time upsampling and rendering framework of algebraic point set surfaces (APSS) [GGG08]. The following table summarizes for both APSS and RIMLS the number of arithmetic operations required by one evaluation as a function of the number of neighbors $k$ and refitting iterations $i$.

\begin{tabular}{c|c|c}
$\begin{array}{c}\text { APSS } \\
\text { (approx normals) }\end{array}$ & $\begin{array}{c}\text { APSS } \\
\text { (exact normals) }\end{array}$ & RIMLS \\
\hline$k * 38+45$ & $k * 130+309$ & $i *(k * 56+13)$
\end{tabular}

\subsection{Analysis of Quality and Stability}

We evaluated the capacity of our approach to reconstruct and preserve surface features on a wide variety of models. 

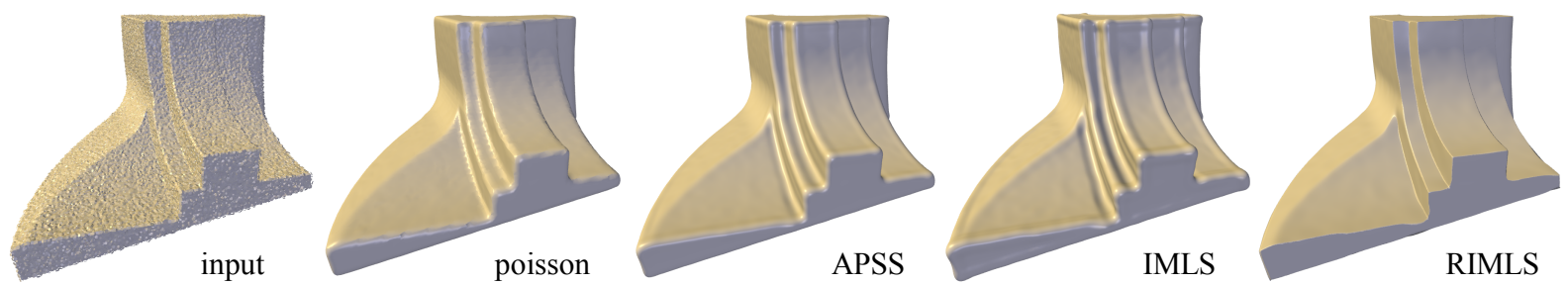

Figure 6: Various reconstructions of the fandisk model after being randomly corrupted by noise of magnitude $0.5 \%$ of the object size.

In the following comparisons, SPSS stands for Adamson et al.'s simple point set surfaces based on normal averaging [AA03].

Moreover, unless stated otherwise, all the results presented here have been produced using the raw normals without using the pre-smoothing procedure described in section 4.4.

In contrast to previous sharp feature reconstruction methods for point set surfaces, we emphasize that the goal of our algorithm is not to generate surfaces with precise normal discontinuities, but rather to improve the faithfulness of the approximation at any scale. This behavior is depicted in figure 8 on a sparsely sampled model. The shape of many parts such as the hands and chest are distorted by previous MLS definitions. In contrast, RIMLS successfully recovers these parts, while smoothing out lower scale details and noise. Sharp features are preserved as can be seen at the corners and edges of the stand, and the tip of the head. The expanding and shrinking effect at the tip of the skirt is also prevented. As can be seen in figure 5, this behavior of RIMLS is also effective in the case of very smooth objects.

The ability of our approach to recover sharp features from sparse sampling is further demonstrated in figure 12. The original model is subsampled to contain approximately $4 \%$

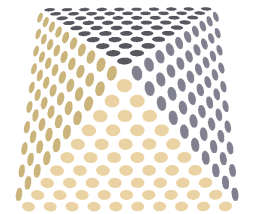

input

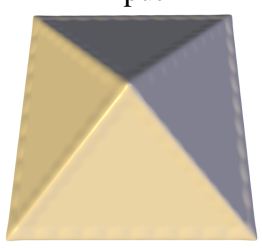

IMLS

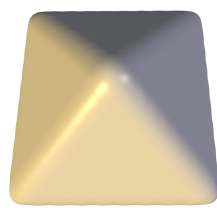

SPSS

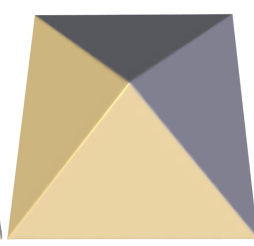

RIMLS (1 iter)

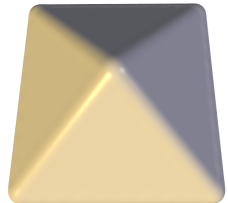

APSS

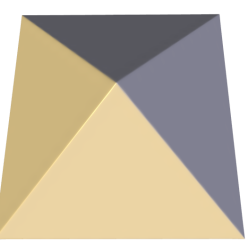

RIMLS (2 iters)
Figure 7: Reconstruction of sharp features for the difficult case of four intersecting planes. of its samples and the resulting model is reconstructed with several MLS definitions. RIMLS preserves fine details and sharp features on the body while providing a more stable overall shape. Note the similarity of the RIMLS reconstruction from the subsampled model to the actual model.

In the same vein, figure 11 includes a comparison to the Poisson surface reconstruction (PSR) technique [KBH06] which also takes into account input normals. While PSR works well in extracting fine details out of densely sampled datasets, when sampling density drops, MLS based approaches, and RIMLS in particular, are clearly superior.

Figure 6 illustrates the ability of our algorithm to reconstruct sharp features from a highly noisy CAD model. As can be seen, because of the large amount of noise, a large spatial filter has to be employed, whence the large undesirable effects produced by both APSS and IMLS near the edges and corners. For the same reason, PSR also produced oversmoothed edges in spite of the relatively dense sampling. Figure 4 demonstrates the stability of the reconstruction under the influence of high noise and outliers. For this difficult case, the advantage of utilizing the pre-smoothing procedure (section 4.4) is evident.

Furthermore, figures 7 and 9 show that our approach can naturally handle high order corners and peaks respectively, which is particularly difficult with previous sharp feature reconstruction methods.

\subsection{Discussion}

Like every functional approximation method, under certain sampling conditions and extreme parameter settings, the quality of the reconstructions can degrade. In particular, if we decrease $\sigma_{n}$ to very small values, there may appear discontinuities in the reconstructions, as can be seen in figure 5 for $\sigma_{n}=0.33$. In fact, when $\sigma_{n} \rightarrow 0$, all samples become outliers of the other leading to a $C^{-1}$ surface. Although we have not done a rigorous theoretical analysis of the convergence and surface continuity with respect to sampling and parameter settings, in our extensive tests we never observed any artifacts for $\sigma_{n}>0.5$, and never feel the need to use a smaller value. As an example, figure 9 includes a rendering with reflection lines showing the high continuity of our surface. 


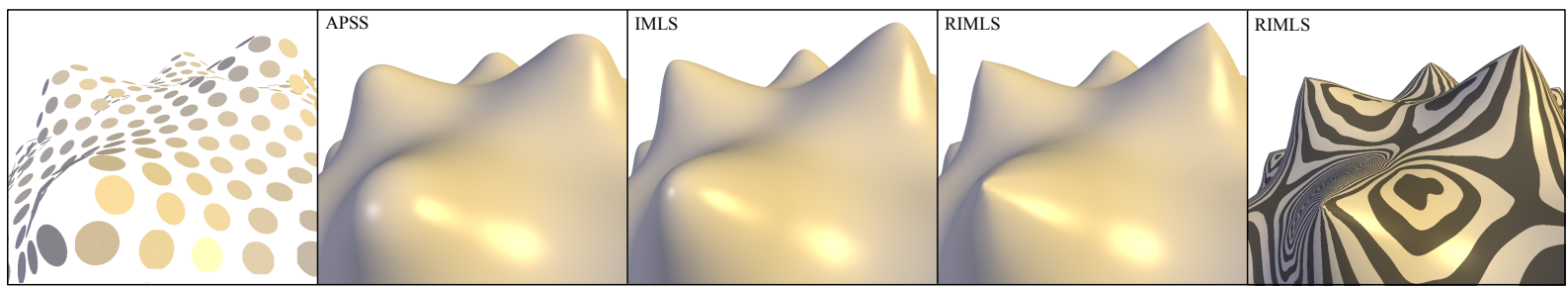

Figure 9: Illustration of the reconstruction of coarsely sampled peaks. Right is a rendering of the RIMLS reconstruction with reflection lines.

\section{Conclusion}

We have demonstrated that MLS and local kernel regression can be advantageously combined to design a novel surface representation with great feature preserving ability. Our approach is particularly robust in the presence of noise, outliers and sparse sampling. While previous sharp feature reconstruction techniques for MLS focused on the generation of exact $C^{0}$ discontinuities at edges and corners, our approach sharpifies the initial over-smoothed solution to a more faithful approximation, keeping the surface differentiable. As a result, our approach can improve the representation of sharp features and fine details of any frequency, and naturally deals with complex situations such as high order corners and peaks. A central advantage of our approach is its extreme simplicity, making it particularly appealing for interactive applications.

Though our results show the reconstructed surface is continuous for a wide range of sampling density and parameters, theoretical bounds on these are yet to be derived in the future.

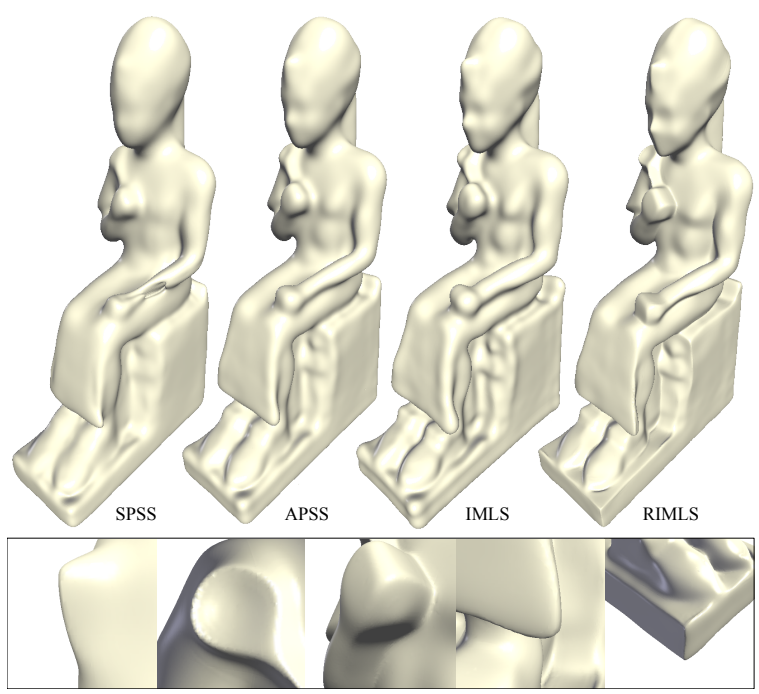

Figure 8: Top row shows a comparison of various MLS definitions on a low sampled Ramses model. Bottom row shows details of the RIMLS reconstruction.
As most of the non-linear techniques, ours depends on the choice of a starting point. While our current solution already provides satisfactory results, we believe it would still be interesting to investigate more robust alternatives.

In addition to the novel surface definition, we showed that MLS based point set surfaces can be interpreted in terms of LKR, suggesting to adopt further methods from regression literature to solve various problems in the geometric setting. By changing the order of regression and constraints, or by investigating unsupervised kernel regression, we believe that superior MLS reconstructions will be possible.

\section{Acknowledgments}

A. Cengiz Öztireli was supported by funding from the Immersion Project. Gaël Guennebaud was supported by the ERCIM "Alain Bensoussan" Fellowship Programme. The
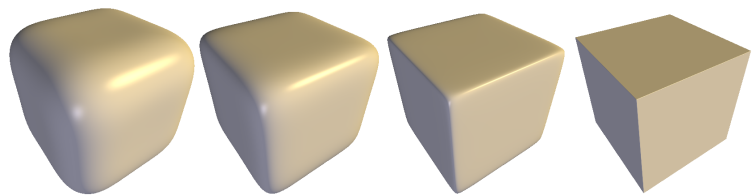

Figure 10: A cube sampled with 4 samples per face reconstructed by RIMLS with $\sigma_{n}=3,1.2,0.75$ and 0.55 .

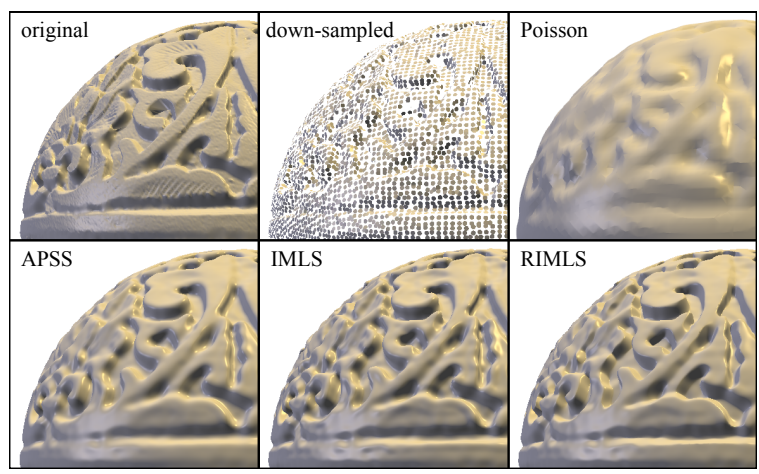

Figure 11: Comparison of various reconstruction methods on a model downsampled to about $2.8 \%$ of the original data. The Poisson reconstruction has been made with a limit of one sample per node and depth 12. 


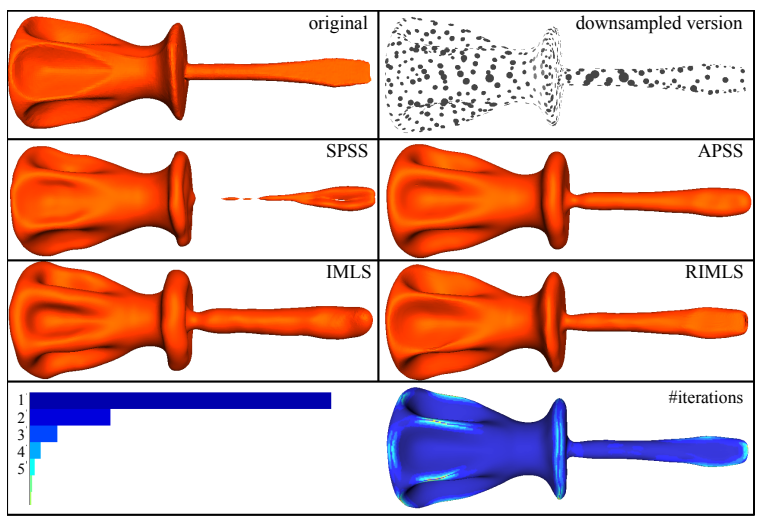

Figure 12: Comparison of different definitions and our definition under very sparse sampling conditions. Bottom row represents the number of RIMLS iterations with the corresponding histogram.

models of figures $1,8,11$, and 12 are courtesy of the AIM@SHAPE Shape Repository.

\section{References}

[AA03] Adamson A., Alexa M.: Approximating and intersecting surfaces from points. In Proceedings of the 2003 Eurographics/ACM SIGGRAPH symposium on Geometry processing (2003), Eurographics Association, pp. 230-239.

[AA04] AleXA M., ADAMSON A.: On normals and projection operators for surfaces defined by point sets. In Proceedings of the Eurographics Symposium on Point-Based Graphics (2004), pp. 149-156.

[AA06a] Adamson A., AleXa M.: Anisotropic point set surfaces. In Afrigraph '06: Proceedings of the 4th international conference on Computer graphics, virtual reality, visualisation and interaction in Africa (2006), ACM Press, pp. 7-13.

[AA06b] Adamson A., Alexa M.: Point-sampled cell complexes. ACM Transactions on Graphics (SIGGRAPH 2006 Proceedings) 25, 3 (2006), 671-680.

[AA07] ALEXA M., ADAMSON A.: Interpolatory point set surfaces - convexity and hermite data. ACM Transactions on Graphics, to appear (2007).

[ABCO*03] Alexa M., Behr J., Cohen-Or D., Fleishman S., Levin D., Silva C. T.: Computing and rendering point set surfaces. IEEE Transactions on Computer Graphics and Visualization 9, 1 (2003), 3-15.

[AK04a] AMENTA N., KIL Y.: Defining point-set surfaces. ACM Transactions on Graphics (SIGGRAPH 2004 Proceedings) 23, 3 (2004), 264-270.

[AK04b] AMENTA N., KIL Y.: The domain of a point set surface. In Proceedings of the Eurographics Symposium on Point-Based Graphics 2004 (2004), pp. 139-147.

[BMdF03] Boris Mederos L. V., De Figueiredo L. H.: Robust smoothing of noisy point clouds. In SIAM Geometric Design and Computing (2003), Nashboro Press, p. 13.

[Cle79] Cleveland W. S.: Robust locally weighted regression and smoothing scatterplots. Journal of the American Statistical Association 74 (1979), 859-836.

(c) 2008 The Author(s)

Journal compilation (c) 2008 The Eurographics Association and Blackwell Publishing Ltd.
[CT03] ChOudhury P., Tumblin J.: The trilateral filter for high contrast images and meshes. In Proceedings of the 14th Eurographics workshop on Rendering (2003), pp. 186-196.

[DHOS07] Daniels J. I., Ha L. K., OсhоTta T., Silva C. T.: Robust smooth feature extraction from point clouds. In $S M I$ '07: Proceedings of the IEEE International Conference on Shape Modeling and Applications 2007 (Washington, DC, USA, 2007), IEEE Computer Society, pp. 123-136.

[FCOS05] Fleishman S., Cohen-Or D., Silva C. T.: Robust moving least-squares fitting with sharp features. ACM Trans. Graph. 24, 3 (2005), 544-552.

[GG07] Guennebaud G., Gross M.: Algebraic point set surfaces. ACM Transactions on Graphics (SIGGRAPH 2007 Proceedings) 26, 3 (2007), 23.1-23.9.

[GGG08] Guennebaud G., Germann M.,, Gross M.: Dynamic sampling and rendering of algebraic point set surfaces. Computer Graphics Forum (Proceedings of Eurographics 2008) 27, 3 (2008).

[GP07] Gross M., Pfister H. (Eds.): Point Based Graphics. Morgan Kaufmann, 2007.

[Hub04] Huber P. J.: Robust Statistics. Wiley, 2004.

[JDD03] Jones T. R., Durand F., Desbrun M.: Noniterative, feature-preserving mesh smoothing. In SIGGRAPH '03: ACM SIGGRAPH 2003 Papers (New York, NY, USA, 2003), ACM, pp. 943-949.

[KBH06] Kazhdan M., Bolitho M., Hoppe H.: Poisson surface reconstruction. In Proceedings of the Eurographics Symposium on Geometry Processing 2006 (2006), pp. 43-52.

[Kol05] KolluRI R.: Provably good moving least squares. In ACM-SIAM Symposium on Discrete Algorithms (Jan. 2005), pp. 1008-1018.

[LCOL07] Lipman Y., Cohen-OR D., Levin D.: Datadependent MLS for faithful surface approximation. In Symposium on Geometry Processing (2007), pp. 59-67.

[Lev03] LEvIN D.: Mesh-independent surface interpolation. $\mathrm{Ge}$ ometric Modeling for Scientific Visualization (2003), 37-49.

[Mor07] Morgenthaler S.: A survey of robust statistics. Statistical Methods and Applications 15, 3 (February 2007), 271293.

[Nad64] NADARAYA E. A.: On estimating regression. Theory of Probability and its Applications 9, 1 (1964), 141-142.

[PKKG03] Pauly M., Keiser R., Kobbelt L. P., Gross M.: Shape modeling with point-sampled geometry. ACM Transactions on Graphics (SIGGRAPH 2003 Proceedings) 22, 3 (2003), 641-650.

[RJT*05] Reuter P., Joyot P., Trunzler J., Boubekeur T., Schlick C.: Point Set Surfaces with Sharp Features. Tech. rep., LaBRI, 2005.

[She68] SHEPARD D.: A two-dimensional interpolation function for irregularly-spaced data. In ACM national conference (1968), pp. 517-524.

[SOS04] SHen C., O'BRIEn J. F., SHewchuk J. R.: Interpolating and approximating implicit surfaces from polygon soup. ACM Transactions on Graphics (SIGGRAPH 2004) (2004), 896904.

[TFM07] TaKeda H., Farsiu S., Milanfar P.: Kernel regression for image processing and reconstruction. IEEE Transactions on Image Processing 16, 2 (2007), 349-366.

[WSS08] Wang H., Scheidegger C., Silva C.: Optimal bandwidth selection for mls surfaces. Shape Modeling and Applications, 2008. SMI 2008. IEEE International Conference on (June 2008), 111-120. 